\title{
Coronary left ventricular outflow tract fistula after resection of a subaortic valve membrane
}

\author{
Stéphane Coutu, MD · Etienne de Médicis, MD • \\ Denyse Normandin, MD · Michel-Antoine Perrault, MD
}

Received: 12 April 2009/Accepted: 7 May 2009/Published online: 1 July 2009

(C) Canadian Anesthesiologists' Society 2009

\begin{abstract}
A 34-yr-old woman was admitted to hospital for resection of a subaortic valve membrane and repair of an ascending aortic aneurysm (Figs. 1 and 2). At 8 months of age, the patient underwent resection of a subaortic valve membrane and closure of a patent ductus arteriosus. On this occasion, the patient underwent resection of the subaortic membrane, ventricular myomectomy, and replacement of the ascending aorta with an aortic valve-sparing technique. At the end of surgery, after unclamping the aorta, a small, central aortic insufficiency grade $1 / 4$ was observed, with no ventricular septal defect. The mitral valve was normal, but an unusual subaortic color Doppler flow was discovered (Fig. 3), which had not been present preoperatively. This color flow was diastolic and appeared to originate from a branch of the left anterior descending (LAD) coronary artery draining into the left ventricular outflow tract (LVOT) just inferior to the aortic valve (Video 1, midesophageal inflow-outflow view). Pulsed-wave Doppler confirmed the typical diastolic flow pattern of the LAD coronary artery (Fig. 4). A fistula from a perforator septal branch of the interventricular septum was presumed to have been responsible for this unusual color flow (also
\end{abstract}

Electronic supplementary material The online version of this article (doi:10.1007/s12630-009-9115-9) contains supplementary material, which is available to authorized users.

S. Coutu, MD $(\varangle) \cdot$ E. de Médicis, MD - M.-A. Perrault, MD Department of Anesthesiology, Centre Hospitalier Universitaire de Sherbrooke, 3001, 12ème Av. Nord, Sherbrooke J1H 5N4, Canada

e-mail: stephane.coutu@usherbrooke.ca

\section{Normandin, MD}

Department of Cardiac Surgery, Centre Hospitalier Universitaire de Sherbrooke, 3001, 12ème Av. Nord, Sherbrooke J1H 5N4, Canada

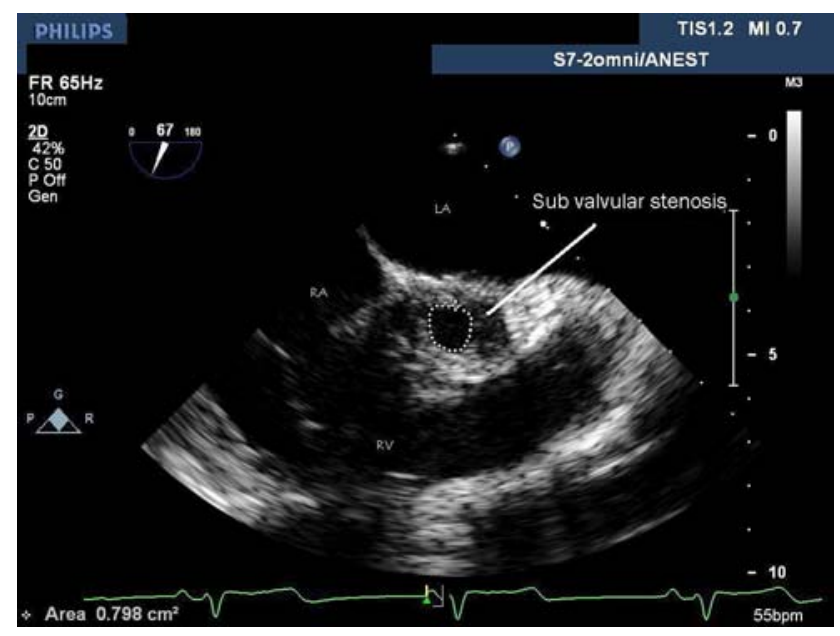

Fig. 1 Mid-esophageal right ventricular inflow-outflow view. A short-axis view of the left ventricular outflow tract (LVOT) is shown. Note the circumferential reduction in the size of the LVOT consistent with subaortic stenosis. $L A$ left atrium; $R A$ right atrium; $R V$ right ventricle

known as a truncated septal perforator). This fistula was not present on the preoperative coronary angiogram. Weaning from bypass was easy and the left segmental ventricular function remained normal. The surgeon decided not to intervene on this new finding, and the patient had an uneventful recovery.

Coronary artery fistulas may be congenital or acquired due to trauma or surgical interventions. These fistulas are quite rare in adults. In a series of 33,600 coronary angiograms in a general population, only $34(0.1 \%)$ were observed, with a good prognosis. ${ }^{1}$ Surgical septal myomectomy may also result in a ventricular septal defect (perimembranous-like ventricular septal defect). In such case, the color flow pattern will be seen between the LVOT and the right ventricle in the mid-esophageal long-axis 


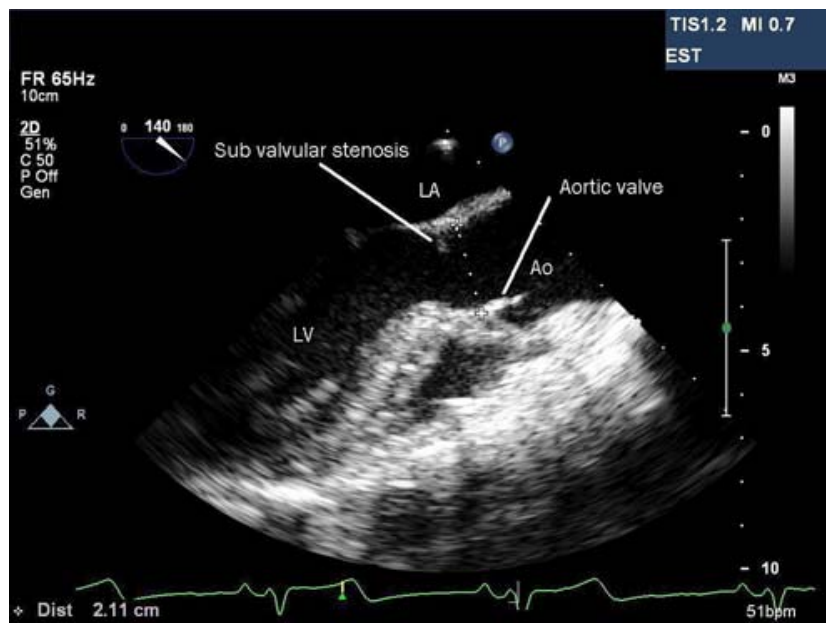

Fig. 2 Mid-esophageal long-axis view of the left ventricle. In the left ventricular outflow tract (LVOT), a membrane is seen consistent with a subaortic stenosis. $L A$ left atrium; $L V$ left ventricle; $A o$ ascending aorta

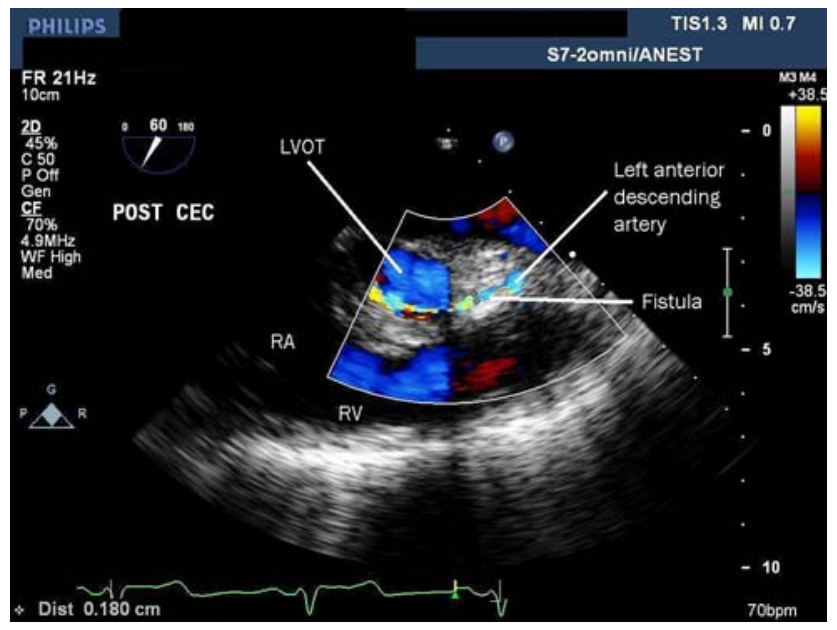

Fig. 3 Mid-esophageal right ventricular inflow-outflow view. A short-axis view of the left ventricular outflow tract (LVOT) is shown. Note the color Doppler flow between the left anterior descending artery and the LVOT, compatible with an iatrogenic coronary-LVOT fistula. $L A$ left atrium; $R A$ right atrium; $R V$ right ventricle; $L V O T$ left ventricular outflow tract

view. Pulsed-wave Doppler will also show a systolic predominant flow towards the right ventricle (in the absence of pulmonary hypertension). In our case, the location and the diastolic flow pattern ruled out a ventricular septal defect. Trauma to the aortic valve can also result in a diastolic color flow and must be considered in the differential diagnosis.

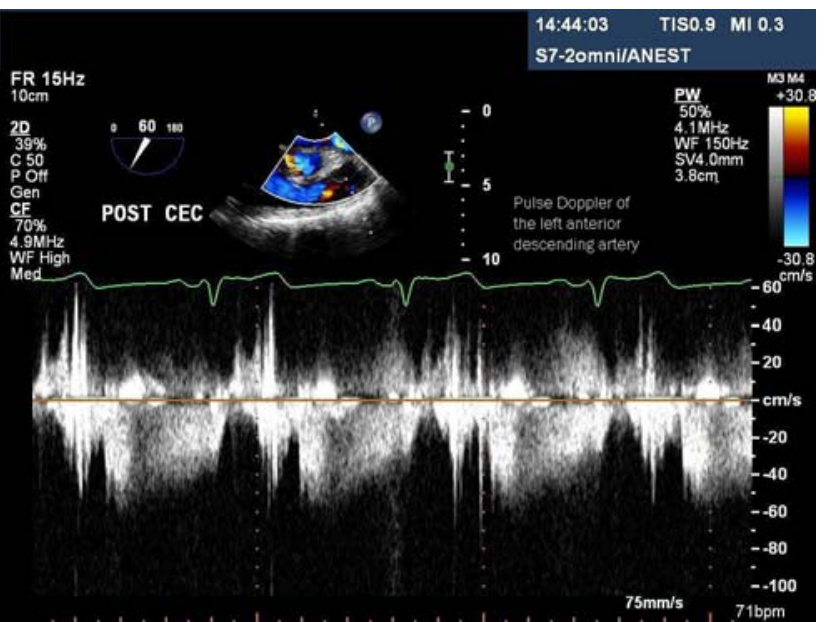

Fig. 4 Pulsed-wave Doppler interrogation of the left anterior descending artery obtained in a mid-esophageal right ventricular inflow-outflow view. Note the diastolic predominance and the typical flow profile (velocity around $40-60 \mathrm{~cm} \cdot \mathrm{sec}^{-1}$ ) of the velocity consistent with a coronary origin

It should be noted that this is a rarely reported occurrence of a coronary-LVOT fistula, ${ }^{2,3}$ although a retrospective series of 26 patients showed a $19 \%$ incidence after ventricular septal myomectomy, ${ }^{4}$ with no clinical consequences. Since our patient was asymptomatic, the decision was taken not to do coronary angiography. The remainder of the postoperative course was uneventful. Although the clinical significance and management of this finding is unknown, this report reinforces the utility of Doppler examination of the coronary circulation in cardiac surgery.

Conflicts of interest None declared.

\section{References}

1. Vavuranakis $M$, Bush CA, Boudoulas $H$. Coronary artery fistulas in adult: incidence, angiographic characteristics, natural history. Cathet Cardiovasc Diagn 1995; 35: 116-20.

2. Jebara VA, Sarkis A, Acar C, et al. Coronary artery-left ventricle fistulas after cardiac surgery. Am Heart J 1991; 122: 1759-62.

3. Sooudi SK, Ahmed S, Nekkanti R, Nanda NC. Transthoracic echocardiographic demonstration of septal perforator coronary arteries-left ventricle fistula. Echocardiography 2003; 20: 197-8.

4. Chenzbraun A, Pinto FJ, Meyer B, Stinson EB, Popp RL. Frequency of acquired coronary-cameral fistula after ventricular septal myectomy in hypertrophic cardiomyopathy. Am J Cardiol 1993; 71: 1244-6. 